\title{
Diffusional limits to the speed of protein folding: fact or friction?
}

\author{
Stephen J Hagen ${ }^{1}$, Linlin Qiu and Suzette A Pabit \\ Physics Department, University of Florida, PO Box 118440, Gainesville, FL 32611-8440, USA \\ E-mail: sjhagen@ufl.edu
}

Received 5 November 2004, in final form 19 December 2004

Published 22 April 2005

Online at stacks.iop.org/JPhysCM/17/S1503

\begin{abstract}
Proteins fold by diffusional motion, driven by molecular collisions but limited by frictional drag. We then expect that the timescale of simple diffusional motions of the polypeptide chain defines the minimum time, or the maximum rate, for folding phenomena in general. However, such 'speed limits' are very rapid. They far exceed the rate of folding that is observed in even the fastestfolding small proteins. Why do proteins fold much more slowly than the diffusional limits predict? We present experimental evidence that, in addition to solvent friction, internal dissipative forces within a protein can slow the dynamics. These internal friction forces may ultimately set a much more restrictive limit on the speed of folding.
\end{abstract}

(Some figures in this article are in colour only in the electronic version)

\section{Introduction}

The study of protein folding dynamics has progressed rapidly in recent years. With the development of new experimental techniques for triggering and probing the fastest folding events, advances in the computational methods for simulating folding, and the discovery (or design) of many new fast-folding proteins and peptides, researchers have found that important steps in the folding pathway can occur on timescales much faster than previously believed possible. Although Anfinsen observed the spontaneous folding of ribonuclease to occur over a period of hours [1], many proteins are now known to make the transition from the unfolded to the native configuration within a few microseconds. At this writing, the fastest folding rates seen in the laboratory approach $\sim 10^{6} \mathrm{~s}^{-1}$ [2-7], a rate that scarcely seemed possible just a few years ago. Many important elementary steps in folding — such as helix formation-occur even faster [8].

This progressive shortening of the 'interesting' timescale for folding raises the question of what sets the physical limit to the speed of protein folding. The rate of diffusional motion

1 Author to whom any correspondence should be addressed. 


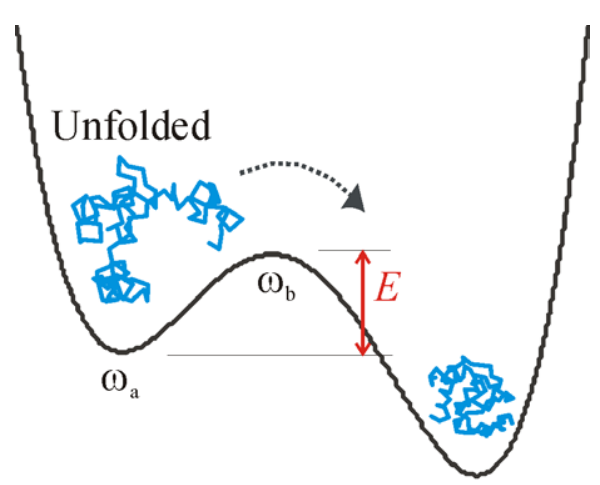

Folded

Figure 1. Two-state, barrier-crossing model for diffusional transition from the unfolded to the folded configuration of a protein $[10,11,28]$. The curvature of the free energy surface is given by $\omega_{\mathrm{a}}$ (for the unfolded states) and by $\omega_{\mathrm{b}}$ at the barrier top.

of a polypeptide chain is an obvious candidate. However, this diffusional 'speed limit' is quite rapid - it is not at all clear that it represents the most stringent upper limit on folding speed. Here I will present evidence that interactions internal to a polypeptide chain — or 'internal friction' phenomena - set a lower and more significant limit on the speed of fast protein folding.

From an experimentalist's perspective, the dynamics of protein folding can often be interpreted as a two-state, barrier crossing process. That is, we can model the transition to the folded state as the passage of a particle, in one dimension, over a free energy barrier that divides the unfolded configurations from the folded state (figure 1). Furthermore, because the dynamics of a polypeptide chain are subject to very strong frictional damping from the solvent, the passage of this particle across the barrier top is driven by Brownian motion. Then there is no 'transition state' as such, and the rate of crossing is not described by the usual transition state theory. Rather it is more appropriately described by the reaction rate theory of Kramers [9-11]. Kramers theory states that, in the limit of strong frictional damping, the particle may diffuse repeatedly across the top of the barrier, but on average it transits the barrier (of height $E$ ) and arrives at the final state at a rate

$$
k=\left(\omega_{\mathrm{a}} \omega_{\mathrm{b}} / 2 \pi \gamma\right) \exp \left(-E / k_{\mathrm{B}} T\right) .
$$

Here $\omega_{\mathrm{a}}$ is the curvature of the unfolded well, $\omega_{\mathrm{b}}$ is the curvature of the barrier top, and $\gamma$ is the frictional drag coefficient that appears in the Langevin equation for the particle's motion. If we interpret $\gamma$ as arising from the dynamic viscosity $\eta_{\mathrm{s}}$ of the solvent, $\gamma \propto \eta_{\mathrm{s}}$, then Kramers yields the unsurprising prediction that $k \propto 1 / \eta_{\mathrm{s}}$, or the folding time for a protein will scale with the solvent viscosity: $k_{\mathrm{f}}^{-1} \propto \eta_{\mathrm{s}}[11]$.

The fact that Brownian motion drives folding suggests that the finite speed of diffusion itself sets the ultimate limit to the speed of protein folding: a protein cannot fold any faster than it can explore (by diffusion) its various configurations [12]. This has led a number of authors to estimate an upper limit on folding speed by measuring the timescale of the simplest reconfigurations of disordered polypeptides. For example, the rate at which end-toend contacts form in a disordered polypeptide has been measured by several groups [13-17]. This rate depends on the number of residues in the loop, but for short loops ( $<10$ residues) it appears to exceed $10^{7} \mathrm{~s}^{-1}$ and may approach $10^{8} \mathrm{~s}^{-1}$. This implies a diffusional 'speed limit' for protein folding rates, of order $k_{\mathrm{f}} \leqslant \sim 10^{8} \mathrm{~s}^{-1}$.

This is a plausible upper limit, if only because no proteins have yet been found to exceed it. In fact, it may be too generous. It exceeds by $\sim 100 \times$ even the very fastest known folding 

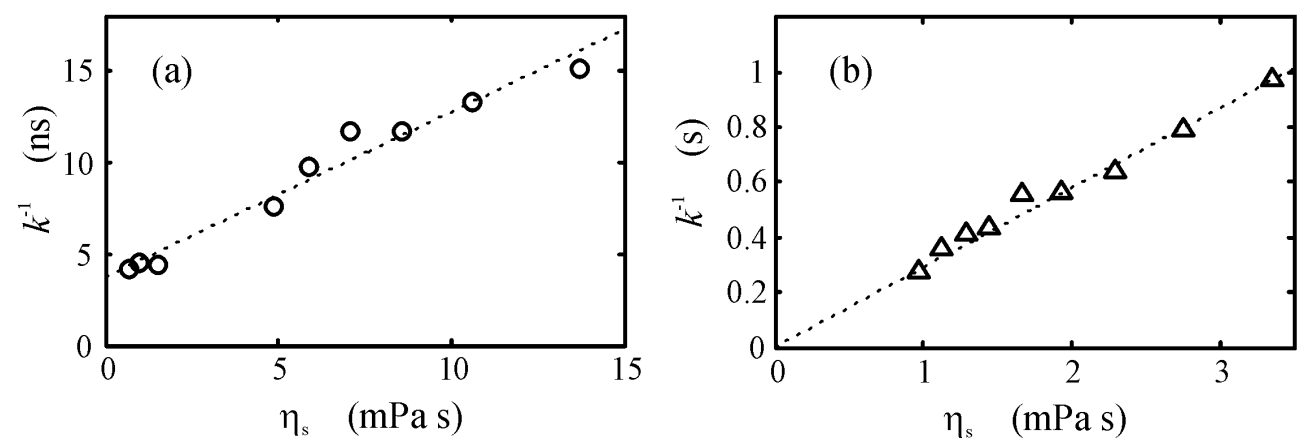

Figure 2. (a) Relaxation time $(1 / k)$ for the conformational relaxation of sperm whale myoglobin, following photodissociation of a haem ligand, adapted from Ansari et al [20]. Extrapolating the dashed line to the horizontal axis indicates $\sigma \approx 4.1 \mathrm{mPa} s$ for this relaxation (equation (2)). (b) Folding time $\left(1 / k_{\mathrm{f}}\right)$ for peptostreptococcal protein $L$ versus solvent viscosity, and linear fit. Data are from Plaxco and Baker [23]. Unlike the myoglobin relaxation data, these folding data are consistent only with a very small $\sigma \approx-0.1 \pm 0.2 \mathrm{mPa}$.

rates. Furthermore, extrapolation from simple correlations, like that between contact order and folding rate [18], suggests that folding rates of small proteins are unlikely to exceed $\sim 10^{6} \mathrm{~s}^{-1}$. Even the collapse of a disordered polypeptide to a molten-globule configuration requires $\sim 10^{-5} \mathrm{~s}[19]$. This raises the question of what other physical phenomena may set more stringent - and therefore more relevant-limitations on protein folding speed. Although early studies suggested otherwise, there is now experimental evidence to suggest that internal friction within a folding protein plays such a role.

\section{Internal friction in protein dynamics}

In 1992, Ansari and coworkers studied a conformational relaxation that occurs in myoglobin after photodissociation of a ligand from the haem iron [20]. They measured the effect of the solvent dynamic viscosity $\left(\eta_{\mathrm{s}}\right)$ on the rate $k$ of this relaxation. Figure 2(a) summarizes their findings: although the relaxation time $k^{-1}$ varies linearly with $\eta_{\mathrm{s}}$ (as expected for a Kramerslike, diffusion-controlled barrier crossing), the relaxation time does not vanish in the limit of low viscosity $\eta_{\mathrm{s}} \rightarrow 0$. Rather, it approaches a finite value $k^{-1}>0$. The authors therefore suggested that, for conformational relaxations deep within a protein, the 'normal' Kramers-like relation $k^{-1} \propto \eta_{\mathrm{s}}$ should be replaced by the empirical formula

$$
k^{-1} \propto \eta_{\mathrm{s}}+\sigma .
$$

That is, the total viscosity contributing to the Kramers friction $\gamma$ is the sum of $\eta_{\mathrm{s}}$ and $\sigma$. Here the extra term $\sigma$ is interpreted as an 'internal viscosity' which strongly limits the speed of the dynamics as the solvent viscosity $\eta_{\mathrm{s}}$ declines. A fit to the data then gives $\sigma \approx 4 \mathrm{mPa}$, indicating that the viscosity of the protein interior is significantly larger than the viscosity of water $\left(\eta_{\mathrm{H}_{2} \mathrm{O}}=1 \mathrm{mPa}\right.$ s at $\left.20.2^{\circ} \mathrm{C}\right)$.

It is then natural to expect that a similar internal viscosity influences the dynamics of protein folding. A number of authors have measured the influence of solvent viscosity on protein folding rates [21-29]. Unfortunately, the viscosity of aqueous solvent cannot be altered without changing other properties of the solvent. At the very least, the addition of viscogenic cosolutes, such as polyols, tends to stabilize proteins through a preferential hydration effect: the exclusion of the cosolute molecules from the immediate environment of the polypeptide chain imposes a large entropic cost on the exposure of the chain to the solvent. This destabilizes 
the unfolded state. The combination of rising viscosity and shifting protein stability may lead to very complicated behaviour of the folding rate.

Some experimental studies have corrected for these effects. Figure 2(b) summarizes the results of Plaxco and Baker [23], who studied the influence of a glucose cosolute on the folding kinetics of peptostreptococcal protein $L$. By simultaneously adding $\mathrm{GdnHCl}$ with glucose, these authors could maintain isostability conditions while raising the solvent viscosity to $\approx 3.5 \mathrm{mPa}$ s. The figure shows that folding time $k^{-1}$ is highly linear in $\eta_{\mathrm{s}}$ in this case, and it extrapolates quite neatly through the origin. The authors reported that the best fit to the Ansari formulation (equation (2)) gives an insignificant internal viscosity $\sigma \approx-0.1 \pm 0.2 \mathrm{mPa}$. A similar puzzling result was also found in several other studies; there was no evidence of a significant internal viscosity influencing protein folding dynamics. Such results are surprising, especially considering that many of the proteins studied fold through highly compact transition states, where internal intrachain interactions would be expected to be significant. If the relaxations of folded myoglobin show such a large internal viscosity, how can the internal viscosity of a protein folding reaction be virtually undetectable?

\section{Origins of internal friction}

The idea that polymer chains are subject to internal frictional forces has a long history in polymer dynamics [30]. Internal friction was originally introduced to explain experimental data on the high frequency rheological and dielectric relaxation properties of polymers. A number of theoretical approaches and models have addressed dissipative mechanisms in polymers that may not directly arise from the solvent viscosity [31]. Manke and Williams [31] point out that essentially all of these arise from the imperfect approximation of describing a polymer chain as a purely elastic or flexible object immersed in a continuum solvent (with a given macroscopic viscosity $\eta_{\mathrm{s}}$ ). Some effects cannot be accurately represented as just a solvent drag, even though they give rise to dissipation or slower dynamics in chain motion. These include the work required to drive backbone bonds across their rotational energy barriers, interactions between side chain atoms, the requirement for void volume to appear in order for the chain to move through a discrete solvent, and potential nonequilibrium effects. As for the application to protein folding, several theoretical studies have implicitly posited a role for internal viscosity in protein folding dynamics [32-34]; however, there remains no clear theoretical prediction for the magnitude of any effect or its possible experimental signature in folding studies. But if many potential mechanisms for internal friction have been proposed in the polymer dynamics literature, one may suspect that the experimental manifestation of internal friction in protein folding could depend on the properties of the folding process, such as whether the molecule is in an expanded or compact configuration, whether one is observing folding versus unfolding, etc. As a simple example, the internal friction resistance that is encountered in pulling apart the endpoints of a long polymer chain is expected to diminish as chain length increases (the 'Kuhn theorem') [30]. By contrast, in compact configurations the friction due to intra-residue side-chain interactions presumably grows with chain length. It is not immediately obvious how one would parametrize internal friction in folding, and-for example-whether the Ansari formulation (equation (2)) is actually appropriate.

An ordinary homopolymer relaxes towards its equilibrium configuration (i.e. towards a random coil) with a relaxation time that is controlled by the solvent viscosity. De Gennes proposed that internal friction would slow this solvent-controlled relaxation time $\tau_{\mathrm{s}}$ by adding a solvent-independent timescale $\tau_{0}$. The relaxation time of the chain, following a perturbation, then becomes

$$
\tau=\tau_{\mathrm{s}}+\tau_{0}
$$


Because $\tau_{\mathrm{s}} \propto \eta_{\mathrm{s}}$, de Gennes suggested that experimental measurements of $\tau$ at several different values of $\eta_{\mathrm{s}}$ would allow one to extrapolate to $\eta_{\mathrm{s}} \rightarrow 0$ and find the internal friction term $\tau_{0}$. (Of course this does not mean that one actually performs experiments in the low friction limit; the extrapolation is the mathematical method for extracting the $\tau_{0}$ contribution under normal solvent, high friction conditions.)

If we apply equation (3) to the time $\tau_{\mathrm{f}}=k_{\mathrm{f}}^{-1}$ for a protein folding reaction, it predicts the same viscosity dependence as does Ansari's equation (2): the folding time should increase linearly with $\eta_{\mathrm{s}}$, with a finite intercept at $\eta_{\mathrm{s}} \rightarrow 0$. The interpretation is very different, however, because equation (3) suggests that a timescale $\tau_{0}$, rather than an internal viscosity $\sigma$, is the appropriate measure of the internal friction effect in protein folding. Folding reactions are rate limited by their slowest step, and if that slow step is strongly solvent-controlled and occurs on a timescale that far exceeds $\tau_{0}$ the contribution from $\tau_{0}$ may not be observable. Although internal friction may prevent a protein from folding in a time less than $\tau_{0}$, this timescale may be so rapid that it limits only the fastest folding phenomena. A very rapid $\tau_{0} \sim$ ns $-\mu$ s would virtually never be detected in kinetics studies on proteins whose folding is limited by slow $(\sim \mathrm{ms}-\mathrm{s})$ processes. Along these lines, the myoglobin data of figure 2(a) and the protein $L$ data of figure 2(b) indicate very different values for $\sigma$, but they are not inconsistent if interpreted, within equation (3), in terms of a very rapid $\tau_{0}$.

\section{Fast folding from a compact state}

This argument suggests that the internal friction should primarily be observable in proteins that fold extremely rapidly, $k_{\mathrm{f}}^{-1} \sim \tau_{0}$, or in the rapid, late stage folding events that follow the slower, large scale reorganization of the polypeptide chain in the solvent. We had the opportunity to study such a late stage process in horse cytochrome $c$ [35]. Roder and coworkers have extensively characterized the equilibrium and kinetic properties of the MCO state of cytochrome $c$, a highly collapsed denatured globule from which folding to the native state can be exceedingly rapid [36-38]. This state is formed by chemically denaturing horse ferrocytochrome $c$, adding carbon monoxide (which binds to the haem iron), and then diluting the denaturant. The dilution causes the protein to collapse to a compact, near folded form, but with the $\mathrm{CO}$ molecule at the haem preventing the formation of the native iron-Met80 ligation. This MCO state therefore possesses largely native-like structure, but with some significant distortion [36]. The molecule is metastable: photodissociation of the $\mathrm{CO}$ from the haem iron allows the molecule to fold to the native state, and it does so within $\sim 10 \mu \mathrm{s}$ at room temperature.

This folding transition is readily monitored through the visible optical absorbance of the haem. Dissociation of the CO from the iron generates a characteristic five-coordinate deoxyhaem absorbance spectrum, which is then replaced by a six-coordinate spectrum as the protein folds and the native methionine ligand reattaches to the haem. We used laser photolysis to trigger the folding, and we monitored its progress through transient absorption spectroscopy [39-41]. The viscosity was adjusted through the addition of glucose, glycerol, or ethylene glycol cosolutes. It is preferable in such studies to use these low molecular weight viscogens because they actually raise the microscopic viscosity experienced by the polypeptide, e.g. slowing processes like ligand escape and rotational diffusion. By contrast, high molecular weight cosolutes (like PEG) appear primarily to affect only the macroviscosity at the laboratory scale, leaving the microscopic dynamics unchanged $[42,43]$.

We directly measured the kinematic viscosity $v$ and density $\rho$ of all samples at all experimental temperatures, and calculated the dynamic viscosity from $\eta_{\mathrm{s}}=v / \rho$. Interestingly, the folding rate of $\mathrm{MCO}$ is insensitive to denaturant concentration up to $\sim 3 \mathrm{M} \mathrm{GdnHCl}$, at which point MCO is itself destabilized in favour of more fully unfolded states. That is, the 


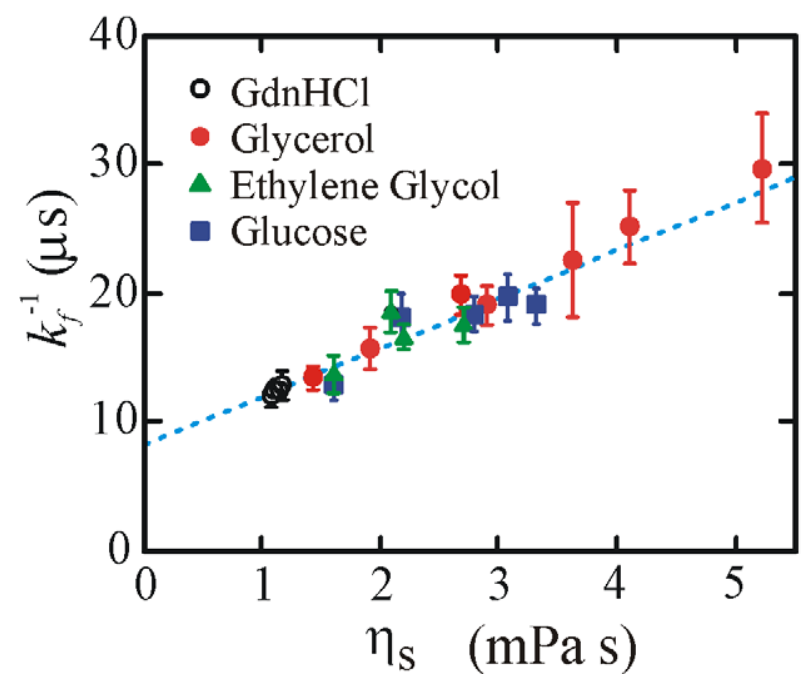

Figure 3. Folding time $\left(1 / k_{\mathrm{f}}\right)$ versus solvent dynamic viscosity $\eta_{\mathrm{s}}$ for horse-heart ferrocytochrome $c\left(\mathrm{pH} 7,20^{\circ} \mathrm{C}\right)$, in the presence of various low molecular weight viscogenic cosolutes. The linear fit extrapolates to a time $8.1 \pm 0.6 \mu \mathrm{s}$ at $\eta_{\mathrm{s}} \rightarrow 0$. The protein is initially in a highly collapsed, denatured configuration (MCO); its folding is triggered optically by photodissociation of the $\mathrm{CO}$ ligand with a 5-7 ns Nd:YAG laser pulse at $532 \mathrm{~nm}$. Folding is probed by transient optical absorbance: after the photolysis flash, a xenon flashlamp and imaging spectrometer project the visible absorbance spectrum onto a gated, intensified CCD camera [35].

dynamics of MCO folding are unaffected by solvent changes that shift the stability of the native state. For this reason no correction was required for stabilizing effects of the viscogenic cosolutes.

A single relaxation is observed in the transient spectra as MCO folds. Because of the overwhelmingly greater stability $\left(17 \mathrm{~kJ} \mathrm{~mol}^{-1}\right)$ of the native state relative to the initial MCO state $[35,36]$, the back unfolding reaction is virtually irrelevant and the time constant extracted from the transient spectra gives the folding rate $k_{\mathrm{f}}$. Figure 3 shows that $k_{\mathrm{f}}$ shows the same behaviour regardless of the choice of viscogen added to the solvent: the folding time $k_{\mathrm{f}}^{-1} \sim 10^{-5} \mathrm{~s}$ increases linearly with $\eta_{\mathrm{s}}$, as expected in a diffusion-controlled Kramers-like process. However, the folding time also clearly extrapolates to a positive value $k_{\mathrm{f}}^{-1} \rightarrow 8.1 \pm 0.6 \mu \mathrm{s}$ in the limit of vanishing solvent viscosity, $\eta_{\mathrm{s}} \rightarrow 0$ at $20^{\circ} \mathrm{C}$. The fact that this limiting rate is barely faster than the rate in aqueous solvent $(\approx 12 \mu$ s) provides clear evidence that the speed of the protein's reorganization is only influenced by-not controlled by - solvent viscosity. Dissipative phenomena arising within the polypeptide evidently play a large role in setting the timescale for folding.

The temperature dependence of this behaviour is even more surprising. Figure 4 shows that $k_{\mathrm{f}}^{-1}$ versus $\eta_{\mathrm{s}}$ remains linear as the temperature of the solvent rises. However the slope does not change greatly. The temperature dependence is dominated by the systematic increase in the $y$-intercept. That is, if we use the simple approach of equation (3),

$$
k_{\mathrm{f}}^{-1}=\tau_{\mathrm{f}}\left(\eta_{\mathrm{s}}, T\right)=\tau_{\mathrm{s}}+\tau_{0}
$$

where $\tau_{\mathrm{s}} \propto \eta_{\mathrm{s}}$, then the internal friction contribution $\tau_{0}$ exhibits a strong temperature dependence that accounts for most of the temperature dependence of the folding rate. This indicates a fairly large scale for the energetics of those interactions that generate the internal friction. A simple Arrhenius fit to the $y$-intercept $\tau_{0}$ (obtained from a linear fit to the data at 


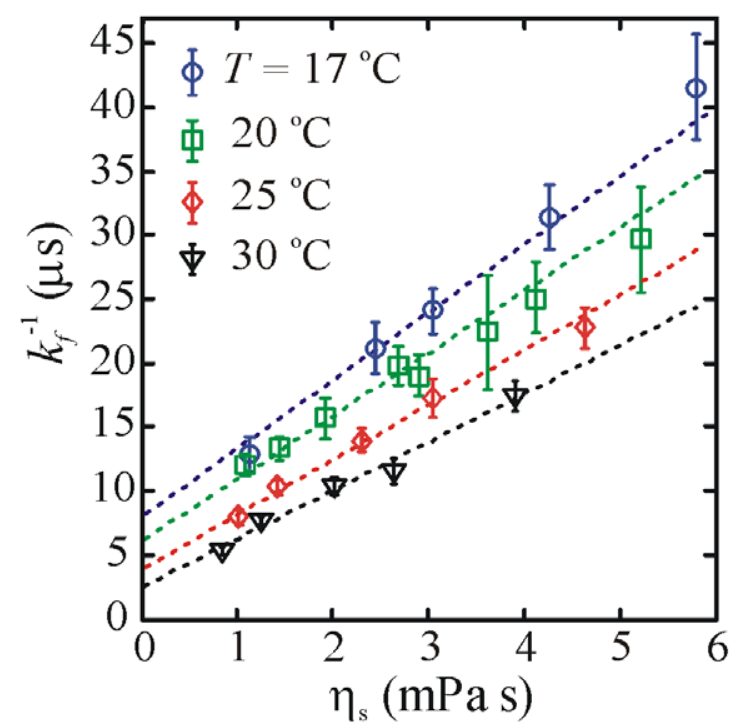

Figure 4. Temperature dependence of folding time $\left(1 / k_{\mathrm{f}}\right)$ for the collapsed state of cytochrome $c$. The dashed lines indicate linear fits to the data at each temperature, in accordance with equation (3), where $\tau_{\mathrm{s}}$ and $\tau_{0}$ are both constrained to an Arrhenius temperature dependence [35]. Because the indicated $\eta_{\mathrm{s}}$ is the dynamic viscosity measured at the indicated temperature, the temperature dependence of solvent viscosity does not contribute to the temperature dependence seen here.

each temperature in figure 4$)$ gives an activation energy $\partial \ln \tau_{0} / \partial\left(1 / k_{\mathrm{B}} T\right) \approx 67 \pm 16 \mathrm{~kJ} \mathrm{~mol}^{-1}$. (Because the abscissa in the figures refers to the actual $\eta_{\mathrm{s}}$ of each sample at the indicated temperature, the temperature dependence of $\eta_{\mathrm{s}}$ itself does not affect the apparent activation energies for $\tau_{\mathrm{s}}$ or $\tau_{0}$.)

The data of figures 3 and 4 show that internal friction can significantly limit the speed of protein folding. They also suggest a very simple explanation for the apparently discrepant results of figures 2(a) and (b): the 'internal viscosity' parameter $\sigma$ suggested by Ansari and coworkers [20] is perhaps not the most useful way to characterize internal friction. It should rather be thought of as setting a lower limit, $\tau_{0}$, to the folding dynamics. This limiting timescale may be present in all protein folding experiments, but it only plays a significant rate-limiting role in proteins that fold on timescales comparable to $\tau_{0}$. Protein $L$ [23] is not such a protein, although cytochrome $c$ is. That is, the protein $L$ and cytochrome $c$ data would give very different values for $\sigma$ in a fit to the Ansari model equation (2), but both experimental studies are consistent with a value $\tau_{0}$ that is of the order of microseconds.

\section{Internal friction in ultrafast folding}

The suggestion that internal friction sets a limiting timescale $(\sim \mu \mathrm{s})$ for folding implies that it could significantly affect the folding of some of the proteins and peptides that have been recently discovered or designed to fold on the 'ultrafast', microsecond timescale. One of the best known examples of such proteins is the tryptophan cage Tc5b (TrpCage), a polypeptide that has been described as the smallest true miniprotein, containing only normal amino acids [44]. TrpCage, only 20 residues in length, contains an alpha helical region, a $3_{10}$ helix, a polyproline II helix, and a compact hydrophobic core: it folds through a two-state transition at a rate $k_{\mathrm{f}}>250000 \mathrm{~s}^{-1}$ at $25^{\circ} \mathrm{C}$ that makes it one of the fastest folding proteins known [2]. 

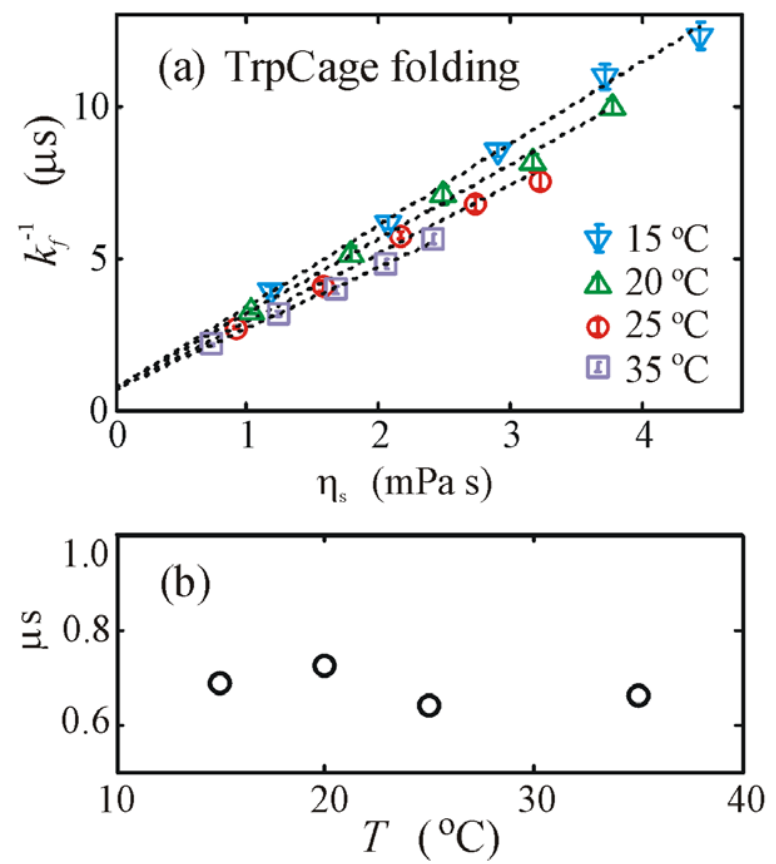

Figure 5. (a) Folding time $\left(1 / k_{\mathrm{f}}\right)$ of the TrpCage Tc5b, as a function of solvent viscosity and temperature, at $\mathrm{pH}$ 7. A linear fit at each temperature is also shown. The thermodynamic stability at each temperature is independent of $\eta_{\mathrm{s}}$. Folding rates are obtained by laser temperature-jump fluorescence spectroscopy, as described previously [2]. A nanosecond laser pulse at $1.06 \mu \mathrm{m}$ is shifted to $1.89 \mu \mathrm{m}$ by stimulated Raman conversion in $\mathrm{H}_{2}$ gas; this shifted pulse causes a rapid (20$30 \mathrm{~ns}$ ) temperature rise in the aqueous solvent of the peptide, triggering re-equilibration between the folded and unfolded configurations of the peptide. These relaxation kinetics are probed by the fluorescence emission of the single tryptophan residue, with $266 \mathrm{~nm}$ laser pulse excitation. (b) Extrapolated value of the folding time at $\eta_{\mathrm{s}}=0$, from the linear fits. The limiting unfolding time is seen to be virtually independent of temperature.

The folding of TrpCage is readily observed through the increase in the fluorescence of the sole tryptophan (W6) upon unfolding. We therefore used laser temperature-jump spectroscopy to trigger folding/unfolding kinetics in TrpCage and measure the effect of solvent viscosity on these kinetics [45].

In this system it is necessary to compensate for stability shifts induced by the viscogen (glucose). Equilibrium experiments established that adding $\mathrm{GdnHCl}$ with the glucose in the ratio $[\mathrm{GdnHCl}] /[$ glucose $] \approx 0.61$ was sufficient to provide isostability conditions over the range of temperatures studied. That is, for each temperature, the folding equilibrium constant $K_{\text {eq }}=k_{\mathrm{f}} / k_{\text {unfold }}$ takes a fixed value, independent of the solvent dynamic viscosity $\eta_{\mathrm{s}}$.

Figure 5 shows the results for $k_{\mathrm{f}}$ versus $\eta_{\mathrm{s}}$. The folding time $k_{\mathrm{f}}^{-1}$ is seen to scale linearly with $\eta_{\mathrm{s}}$ over the entire range of viscosities studied. In fact, although $k_{\mathrm{f}}$ for TrpCage is strongly temperature dependent, plotting the folding time against the solvent viscosity almost causes the data for different temperatures to overlap. That is, the folding rate of any given sample of TrpCage varies strongly with temperature, but this variation largely disappears when the rates at different temperatures are compared at fixed solvent viscosity. Folding in this system therefore appears diffusion controlled, and lacks any enthalpic barrier; it is largely an entropic search. Further, as in the folding of the compact denatured state of cytochrome $c$ we find that $k_{\mathrm{f}}^{-1}$ extrapolates to a small but clearly nonzero limiting value as $\eta_{\mathrm{s}} \rightarrow 0$. As in the compact 

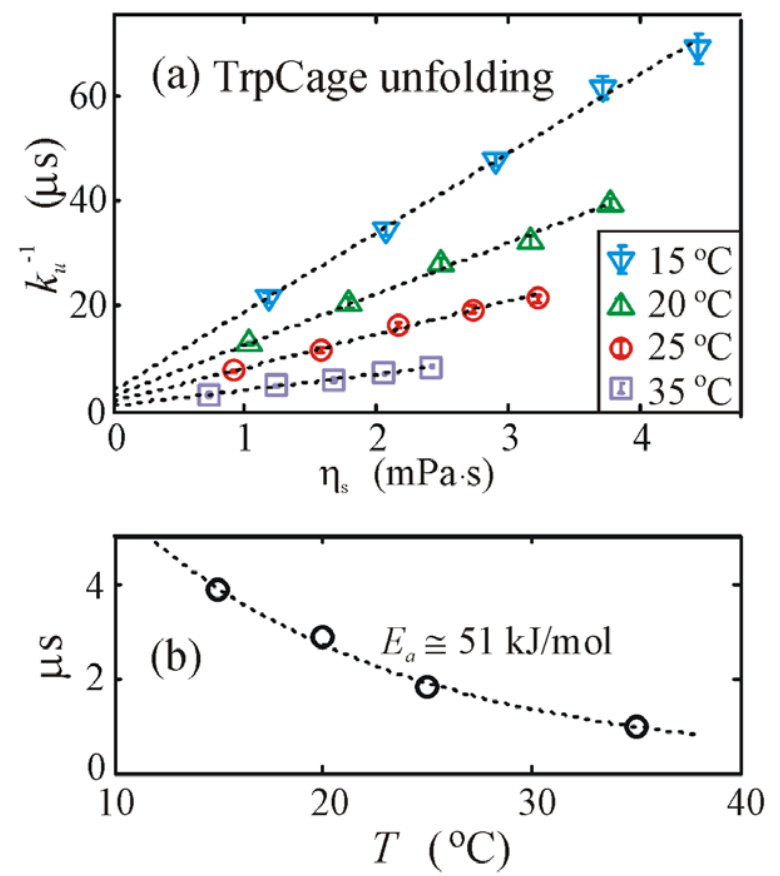

Figure 6. (a) Unfolding time ( $\left.1 / k_{\mathrm{u}}\right)$ of TrpCage, with linear fit at each temperature. (b) Extrapolated value of $1 / k_{\mathrm{u}}$ at $\eta_{\mathrm{s}}=0$, from the linear fits. The dotted curve shows an Arrhenius fit to the data, giving an activation energy $\approx 51 \mathrm{~kJ} \mathrm{~mol}^{-1}$ for the limiting unfolding time of TrpCage.

cytochrome $c$, the folding dynamics approach a finite limiting speed at low solvent viscosity. This provides further evidence that internal friction influences protein folding, although the limiting timescale for TrpCage is significantly smaller than for the MCO state of cytochrome c. Fitting the data of figure 5(a) to a straight line at each temperature gives the $y$-intercept or $\tau_{0}$ values shown in figure $5(\mathrm{~b})$; these are of order $0.7 \mu$ s for all measurements over the temperature range $15-35^{\circ} \mathrm{C}$.

Therefore we find that the folding times of both the 20-residue TrpCage and the 104residue MCO state of cytochrome $c$ approach a finite limiting value at low solvent friction. The internal friction in these two systems differs however in its temperature dependence: a strongly activated $\tau_{0}$ in the compact globule MCO is replaced by a $T$-independent $\tau_{0}$ in the smaller TrpCage. This is not entirely unexpected. As suggested above, the existence of many conceivable physical mechanisms for internal friction in folding suggests that this could be seen as a class of phenomena, which could appear different in different experimental systems.

As evidence for this view, consider the unfolding rate $\left(k_{\mathrm{u}}\right)$ data for TrpCage (figure 6(a)). As with folding, the unfolding time scales with solvent viscosity. It also extrapolates towards a finite value $k_{\mathrm{u}}^{-1} \sim 1-4 \mu$ s in the limit of low solvent viscosity. However, this limiting timescale for unfolding varies strongly with temperature; an Arrhenius fit to the intercept $\tau_{0}(T)$ implies a fairly large activation energy of $51 \mathrm{~kJ} \mathrm{~mol}^{-1}$.

Finally, it is quite interesting to note recent molecular dynamic simulations of the viscosity dependence of TrpCage folding [46]. Simulations allow the 'experimenter' to explore the regime $\eta_{\mathrm{s}} \ll 1 \mathrm{mPa}$ s that is inaccessible in the laboratory. Figure 7 shows that Zagrovic and Pande observed a deviation from simple $k_{\mathrm{f}} \sim 1 / \eta_{\mathrm{s}}$ behaviour at low $\eta_{\mathrm{s}}$. However, this is a different kind of deviation from that behaviour than appears in the laboratory data. The 


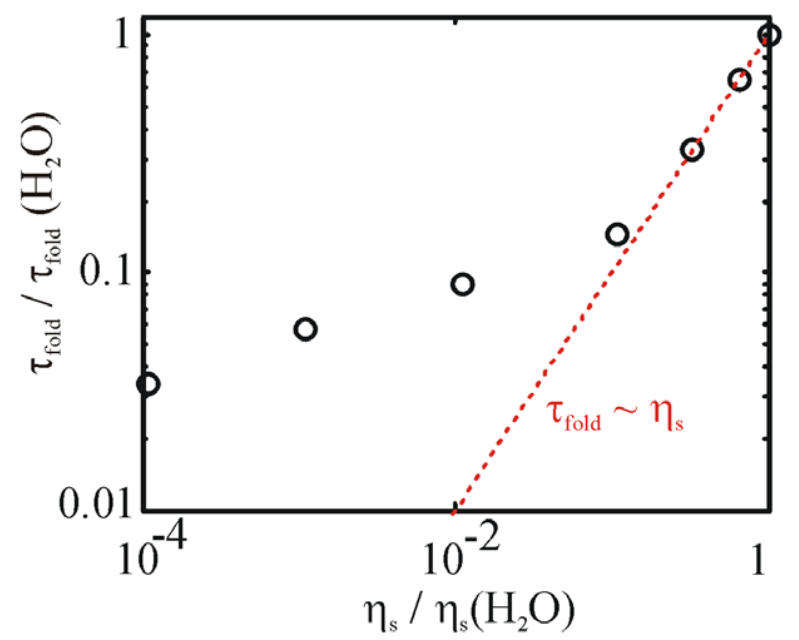

Figure 7. Folding time of TrpCage versus solvent viscosity, from molecular dynamics simulation. The dashed line indicates the behaviour for a simple Kramers rate, $k_{\mathrm{f}}^{-1} \sim \eta_{\mathrm{s}}$. Data are from [46].

origin of this discrepancy is unknown. One may speculate that it is associated with the implicit or continuum solvent approximation that was used in the simulation studies. This kind of experimental/simulation comparison may shed light on the microscopic origin of internal friction, and it will likely be an interesting direction for future studies.

In summary, the TrpCage data show that, for an ultra-fast folding protein, folding and unfolding are not necessarily controlled only by the viscosity of the solvent. The response of these rates to changes in $\eta_{\mathrm{s}}$ suggests that additional, internal friction mechanisms within the protein dissipate energy from the reaction coordinate during folding. Internal friction does play a role in fast protein folding dynamics.

\section{Conclusions}

The evidence for internal friction in TrpCage, when none was observed in protein $L, C s p B$, or several other slower folding systems, supports the hypothesis that internal friction could set a limiting timescale for folding phenomena. We suggest that the phenomenon will be most evident - in fact it will perhaps only be visible - in the fastest folding dynamics. Based on the data currently available for cytochrome $c$ and TrpCage, the relevant timescale appears to be of order $\sim \mu$ s. This is of course the same order of magnitude as the very fastest known folding rates. However, the roughly tenfold difference in the magnitude of the internal friction parameter $\tau_{0}$ for the two proteins studied here-and the differences in the temperature dependence of $\tau_{0}$ - suggest that internal friction could arise from different mechanisms in different systems, or in different types of dynamics, or in different scenarios of folding versus unfolding.

Some elementary steps in folding show evidence of internal friction effects. Deviations from a simple Kramers relation, $k \sim 1 / \eta_{\mathrm{s}}$ were observed in the kinetics of formation of an $\alpha$-helix $(\sim$ ns $)$ but not in the formation of a $\beta$-hairpin $(\sim \mu \mathrm{s})$ [47]. Similarly, in a study of the rate of formation of intrachain, end-to-end loops in disordered polypeptides as a function of solvent viscosity, the rate of this relatively simple process was found to exhibit an 'excess' temperature dependence, beyond that associated with the solvent viscosity $\eta_{\mathrm{s}}(T)$ [48]. This is consistent with the idea within some internal friction models that even simple reconfigurations of a polypeptide backbone require thermal activation across energetic barriers. 
Overall the internal friction-limited folding rates seen here, $\sim 10^{6} \mathrm{~s}^{-1}$, are comparable to the very fastest protein folding rates that have been observed to date. They are also consistent with estimates for the maximum possible rates that would be derived empirically from contact order and other such experimental correlations. However they fall far short of the very rapid rates $\sim 10^{7}-10^{8} \mathrm{~s}^{-1}$ observed for simple diffusional processes in simple chains. This suggests that the diffusional 'speed limits' to protein folding, which consider only the free motion of a disordered polypeptide chain, may rather substantially overestimate the upper limits to protein folding speed. It will be interesting to see whether experimental studies can further clarify and characterize internal friction mechanisms. At the simplest level, for example, is there a simple chain length dependence of $\tau_{0}$ ? If so we may expect that shorter proteins may ultimately be capable of faster folding. Is there a more appropriate way to measure an 'internal friction parameter' for a protein folding reaction? These questions will become increasingly important as increasing numbers of small, ultrafast folding proteins are designed and discovered.

\section{Acknowledgments}

$\mathrm{SJH}$ is grateful to Professor Marek Cieplak for the invitation to participate in the Będlewo meeting. Funding support from the National Science Foundation, through MCB 0077907 and MCB 0347124, is also gratefully acknowledged. The authors thank H Roder for his collaboration in the cytochrome $c$ study.

\section{References}

[1] Anfinsen C B, Haber E, Sela M and White F H 1961 Proc. Natl Acad. Sci. USA 471309

[2] Qiu L L, Pabit S A, Roitberg A E and Hagen S J 2002 J. Am. Chem. Soc. 12412952

[3] Zhu Y, Alonso D O V, Maki K, Huang C Y, Lahr S J, Daggett V, Roder H, DeGrado W F and Gai F 2003 Proc. Natl Acad. Sci. USA 10015486

[4] Arora P, Oas T G and Myers J K 2004 Protein Sci. 13847

[5] Yang W Y and Gruebele M 2004 Biophys. J. 87596

[6] Wang T, Zhu Y J and Gai F 2004 J. Phys. Chem. B 1083694

[7] Yang W Y and Gruebele M 2003 Nature 423193

[8] Eaton W A, Munoz V, Hagen S J, Jas G S, Lapidus L J, Henry E R and Hofrichter J 2000 Annu. Rev. Biophys. Biomol. Struct. 29327

[9] Kramers H A 1940 Physica 7284

[10] Hanggi P, Talkner P and Borkovec M 1990 Rev. Mod. Phys. 62251

[11] Klimov D K and Thirumalai D 1997 Phys. Rev. Lett. 79317

[12] Kubelka J, Hofrichter J and Eaton W A 2004 Curr. Opin. Struct. Biol. 1476

[13] Hagen S J, Hofrichter J, Szabo A and Eaton W A 1996 Proc. Natl Acad. Sci. USA 9311615

[14] Hagen S J, Hofrichter J and Eaton W A 1997 J. Phys. Chem. B 1012352

[15] Bieri O, Wirz J, Hellrung B, Schutkowski M, Drewello M and Kiefhaber T 1999 Proc. Natl Acad. Sci. USA 96 9597

[16] Lapidus L J, Eaton W A and Hofrichter J 2000 Proc. Natl Acad. Sci. USA 977220

[17] Krieger F, Fierz B, Bieri O, Drewello M and Kiefhaber T 2003 J. Mol. Biol. 332265

[18] Plaxco K W, Simons K T and Baker D 1998 J. Mol. Biol. 277985

[19] Qiu L L, Zachariah C and Hagen S J 2003 Phys. Rev. Lett. 90168103

[20] Ansari A, Jones C M, Henry E R, Hofrichter J and Eaton W A 1992 Science 2561796

[21] Chrunyk B A and Matthews C R 1990 Biochemistry 292149

[22] Jacob M, Schindler T, Balbach J and Schmid F X 1997 Proc. Natl Acad. Sci. USA 945622

[23] Plaxco K W and Baker D 1998 Proc. Natl Acad. Sci. USA 9513591

[24] Bhattacharyya R P and Sosnick T R 1999 Biochemistry 382601

[25] Jacob M and Schmid F X 1999 Biochemistry 3813773

[26] Jacob M, Geeves M, Holtermann G and Schmid F X 1999 Nat. Struct. Biol. 6923

[27] Ladurner A G and Fersht A R 1999 Nat. Struct. Biol. 628

[28] Bilsel O and Matthews C R 2000 Adv. Protein Chem. 53153 
[29] Silow M and Oliveberg M 2003 J. Mol. Biol. 326263

[30] de Gennes P G 1979 Scaling Concepts in Polymer Physics (Ithaca, NY: Cornell University Press) p 324

[31] Manke C W and Williams M C 1985 Macromolecules 182045

[32] Portman J J, Takada S and Wolynes P G 2001 J. Chem. Phys. 1145082

[33] Chan H S, Shimizu S and Kaya H 2004 Methods Enzymol. 380350

[34] Kaya H and Chan H S 2002 J. Mol. Biol. 315899

[35] Pabit S A, Roder H and Hagen S J 2004 Biochemistry 4312532

[36] Latypov R F, Maki K, Cheng H, Luck S D and Roder H 2003 J. Mol. Biol. at press

[37] Maki K, Latypov R F, Cheng H, Luck S D, Pabit S A, Hagen S J and Roder H 2003 J. Mol. Biol. at press

[38] Bhuyan A K and Kumar R 2002 Biochemistry 4112821

[39] Hagen S J, Latypov R F, Dolgikh D A and Roder H 2002 Biochemistry 411372

[40] Hagen S J, Carswell C W and Sjolander E M 2001 J. Mol. Biol. 3051161

[41] Jones C M, Henry E R, Hu Y, Chan C K, Luck S D, Bhuyan A, Roder H, Hofrichter J and Eaton W A 1993 Proc. Natl Acad. Sci. USA 9011860

[42] Yedgar S, Tetreau C, Gavish B and Lavalette D 1995 Biophys. J. 68665

[43] Lavalette D, Tetreau C, Tourbez M and Blouquit Y 1999 Biophys. J. 762744

[44] Neidigh J W, Fesinmeyer R M and Andersen N H 2002 Nat. Struct. Biol. 9425

[45] Qiu L L and Hagen S J 2004 J. Am. Chem. Soc. 1263398

[46] Zagrovic B and Pande V 2003 J. Comput. Chem. 241432

[47] Jas G S, Eaton W A and Hofrichter J 2001 J. Phys. Chem. B 105261

[48] Lapidus L J, Steinbach P J, Eaton W A, Szabo A and Hofrichter J 2002 J. Phys. Chem. B 10611628 